\title{
The Hierarchical Order of Spaces in Arab Traditional Towns: The Case of Najd, Saudi Arabia
}

\author{
Mohammed Mashary Alnaim \\ Department of Architectural Engineering, Faculty of College of Engineering, University of Hail, Hail, Saudi Arabia \\ Email: moha.alnaim@gmail.com
}

How to cite this paper: Alnaim, M.M. (2020) The Hierarchical Order of Spaces in Arab Traditional Towns: The Case of Najd, Saudi Arabia. World Journal of Engineering and Technology, 8, 347-366. https://doi.org/10.4236/wjet.2020.83027

Received: June 20, 2020

Accepted: July 21, 2020

Published: July 24, 2020

Copyright $\odot 2020$ by author(s) and Scientific Research Publishing Inc. This work is licensed under the Creative Commons Attribution International License (CC BY 4.0).

http://creativecommons.org/licenses/by/4.0/

\begin{abstract}
The built environment faces many challenges in accommodating multi-cultural diversity and migrating from the suburbs, which led to different places being isolated or neglected. Sub-identities established by a specific group of people become isolated and away from the shared identity that the built environment established for itself. We examine this issue in the Najdi built environment by examining how Najdi locals generated a holistic hierarchical order of specs. This paper argues that the spatial order encouraged locals to develop a shared and agreed mechanism while in parallel, it gave individuals the ability to modify their places without affecting that shared holistic spatial order.
\end{abstract}

\section{Keywords}

Hierarchy, Order, Space, Built Environment, Najd, Urban Fabric, Culture, Space Syntax

\section{Introduction}

To understand the structure of the place, it is essential to distinguish between "settlement" and "character." The settlement is a creation of spaces. Spaces establish the relationship between inside and outside, which both imply that spaces possess a varying degree of enclosure and openness. The character of a place is viewed as the general atmosphere which is the most comprehensive property of any place (i.e., architectural elements), which makes the character of a place an articulation tool for how things are made in any place [1]. It is important to view the place both through space (i.e., the two-dimensional organization of spaces) as well as through character (the three-dimensional organization of the elements 
which establish a place). Thus, the form and its space constitute primary elements of architecture.

In this sense, the place is the overall phenomenon that produces space and character by people to express their differences while integrating their cultural settings and environmental conditions. This means that place is a field of social actions and influences how to construct and contest, and usually establishes personal experience [2]. The emphasis on the importance of tracing the meaning of place in order to understand the process of making of place is mostly a shared and agreed view [3] [4] [5] [6].

Understanding the process that made a place the way and the shape it enables the recognition and understanding of the different associated meanings in a place and why a place expresses different shapes and forms in particular ways. In this sense, one can see the process of placemaking as an aspect of people's reaction to the place. One understands these reactions by examining the privacy and specific behaviors that influenced the association or segregation of organizational spaces in the place. For instance, the separation between male and female spaces, which classifies the spaces into distinct zones within the place, influences the structure of the place. Further, the layout of a pedestrian pavement (or how the pavement is not laid out) in support of how a community uses the space which will eventually impose new ones that are a reaction by the community to support their way of use. This can happen in different ways, from culture to culture, which can produce different places.

In this paper, we examine five Najdi traditional cases: Ad-Diriya, Sudus, Alkhabra, Ushaiqer, and old Riyadh to observe and examine four phases related to the hierarchal order of spaces analysis. The first phase is to understand the process. The first phase is to understand the process in the built environment that generated the hierarchal order of spaces. The second phase is to examine the different street types from the most public to the most private to identify the mechanism of the hierarchal order of spaces. The third phase is to explore the external space patterns of number of Najdi cases. The fourth phase is to conclude with shapes and forms of external streets.

\section{Understanding the Concept of Hierarchy in a Built Environment}

Reference [7] discusses the issue of "abandon anomique" where loss of social norms and control leads to the place to be "incoherent". Two cognitive tools presented that help comprehend the built environment: the first is "microsocial", where certain places and spaces are the values that formulate the urban sphere. The second is "experience", where places and spaces gain significant value from the social actions and connections that people generate within the place and space [7]. Both tools provide an understanding of the built environment through observation/reflection.

This is why on examining the built environment we found that the decisions made by the inhabitants in their private neighborhoods we suggest do strongly 
influence the hierarchical order of spaces. This hierarchy is generated to limit the "abandon anomique" and try to organize the built environment in a more "coherent" way. Even though larger public domain are significant, certain constraints at the micro level, such as socio-cultural needs, privacy, security, etc., have priority in producing the hierarchal order of spaces in certain cultures (Figure 1). At the micro-level, people engage in the process which affects the urban fabric production in different ways, such as how dense the hellas (neighborhood/urban mass) are in terms of number of buildings, how streets are arranged and connected with public and semi-public spaces, the ratio of the semi-private open spaces in relation to hella urban mass, etc. We argue that this directed the process of generating the spatial and physical forms at the macro-level, which influenced and organized the connectivity and integration of different private urban masses (hellas), public open spaces, and the locations of public buildings.

Any decision-making differences observed between the micro and macro levels do not necessarily mean that they are completely different, but rather the micro and macro level decisions work in parallel from two different directions to produce a homogenized built environment. One is to organize the spatial order based on shared socio-cultural values. The second is to give inhabitants the ability to produce their spatial order based on their needs while preserving the shared and agreed upon socio-cultural meanings embodied in the spatial hierarchy.

This brings the paper discussion to a broader question, which is: "who owns the street at the micro-level in the traditional Najdi built environment?" Several researchers investigated this issue in the Arabic-Islamic traditional built environment context and concluded that the inhabitants usually own and control the streets and any other public spaces collectively, they are not owned by the ruler [8] [9] [10]. This is important as it means that there should be a mechanism of agreement among people to enable them to produce, reproduce and manage external spaces in their built environment. This mechanism works on two levels: the collective and sub-collective levels. The collective level is what is meant by macro level (society) and works on external spaces shared by the entire settlement. The sub-collective level (i.e. extended family) is at the micro-level and mainly works on external spaces shared by the private neighborhood and housing clusters.

\section{The Generative Process in the Hierarchical Order of Spaces}

Examining external streets in the Najdi built environment led to understand that two types of streets appeared in the traditional Najdi built environment: public streets and semi-private pathways. The main principle applied to the decisions related to these two types of streets is that any actions related to the pathways are taken by the inhabitants of the hella (sub-collective), while actions related to the public streets are taken by the local inhabitants collectively. This suggests that 


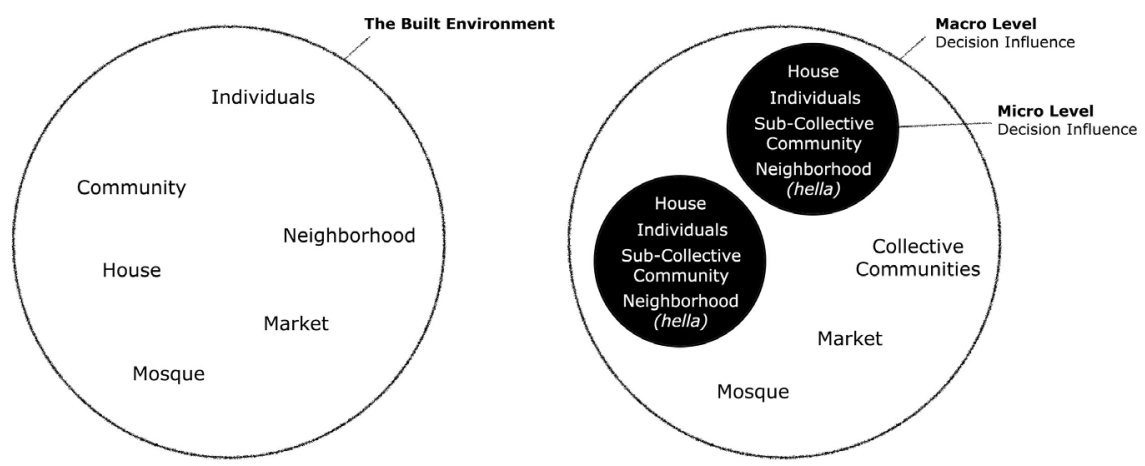

Figure 1. Macro and micro levels in the traditional built environment. Source: Author.

various pathways in different residential parts of the settlement have different formations depending on how various groups in the hellas manage to make the right decision for their particular circumstances. This also suggests that the more streets that belong to the public domain the less change will occur. This limitation on change is because public streets fall in the larger social circle which requires collective agreement and decision by the community to approve any change.

Reference [8] discusses that streets are the most susceptible to change in the traditional built environment by studying similar cases [8]. This paper argues that the spatial order influences the production of the built form in a way that maintains a level of homogeneity between the macro (settlement) level and the micro (private neighborhood) level, or from another, more granular perspective, between the public, semi-private and private domains. This means that any new change passes through several formal and/or informal review processes and decisions, and finally the proposed change is either approved or discarded. The final decision is judged by considering the principle of "no harm" according to the religious principle of "no harm or damage" which guarantees that any action taken in the built environment should not harm any of its residents ${ }^{1}$. In fact, this principle gives incredible freedom for anyone to act and change the built environment as long as his action will not harm anyone. This principle maintains the level of homogeneity that the original spatial order produced, because it guarantees freedom of action of the inhabitants, but within the limitation of the "no harm or damage" principle which allows the community to prohibit or modify harmful actions.

Reference [11], for example, reports a case from Medina (1268/1852) in which a group of people sued a neighbor who closed their pathway by extending part of his house across it, thus transforming the pathway into two cul-de-sacs. The neighbors won the case by proving that the action will negatively affect the behavior of inhabitants and negatively affects the composition of the street's usage which therefore harms the community [11]. Similarly, reference [12] reports a case in Ushaiqer of an individual who wanted to protrude parts of his house into "No harm, no damage" is a prophetic saying and is used widely in Islamic law to evaluate people's actions toward others and it is still a regulatory process used today. 
a street. In this case, the affected users of the street testified that the street belongs to the public right-of-way, and the action will negatively affect its usage. By majority agreement, the proposed action was rejected [12]. In this sense, different users view actions from different perspectives, which refines the process of generating and regenerating the built form.

Reference [9] examines street classification in the traditional built environment according to the Fuqah (Islamic jurisprudence). He concludes that streets are exposed to concepts and elements while produced, such as safety, privacy, accessibility, etc., to ensure public right-of-way [9] ${ }^{2}$. Therefore, as a result of this study and with the support of other mentioned studies, a set of principles and questions is proposed and developed that should be considered by anyone in the process of producing a hierarchal order of spaces. These principles and questions are meant to protect the occupant's rights and perceive the street as having an element of co-ownership (Figure 2).

During these daily actions and decisions, people usually associate their hidden meanings with the spatial and physical forms around them. This study noted that people's interactions in the traditional built environment are one of the main mechanisms for transmitting social values to the built forms. The agreement and disagreement between people take the built environment to a level of balance that produces collective actions or at least agreed upon actions by the community. Through these daily interactions, the values, preferences, and experiences of inhabitants become embedded in the spatial and physical forms.

The above discussion highlighted the process that arranges the spaces in the traditional Najdi built environment. The concluded examination indicates that certain religious principles govern people's actions and interactions and control their daily decisions. These principles include Alshuf a ${ }^{3}, H_{a q}$ Alirtifa $q^{4}$, and the overarching principle of "no harm". They function according to people's interpretations and agreements in each settlement. This produced, on the one hand, different shapes across the Najd settlements for urban masses, open spaces, and streets, while on the other hand, the principles unified the use and meanings of these urban elements across those same settlements.

\section{External Space Types}

The previous discussion concluded that the hierarchal order of spaces generates and classifies streets into two types: public and semi-private. Each type, while different social domains produce and control it, still shares the same hierarchical ${ }^{2}$ An interview with Basim Hakim in Albuquerque, New Mexico on 22/03/2017 was also conducted to gain more insight on his approach for how religious values can have a role in the production of the built environment.

${ }^{3}$ Alshuf $a$ is an Islamic law which controls the buying and selling among people. The Shuf a principle from this perspective, seeks to preserve the interest among different owners, ensuring that "no harm comes to the partner". If many partners are all involved in this right, it is not permissible to sell to one of them without the rest.

${ }^{4}$ The "easement right" is the benefit of a property, such as drinking (from a well), traffic (street), land, etc., located within an owned personal property that he does not own, but is owned by another person or owned by the entire local built environment. See more [13]. 


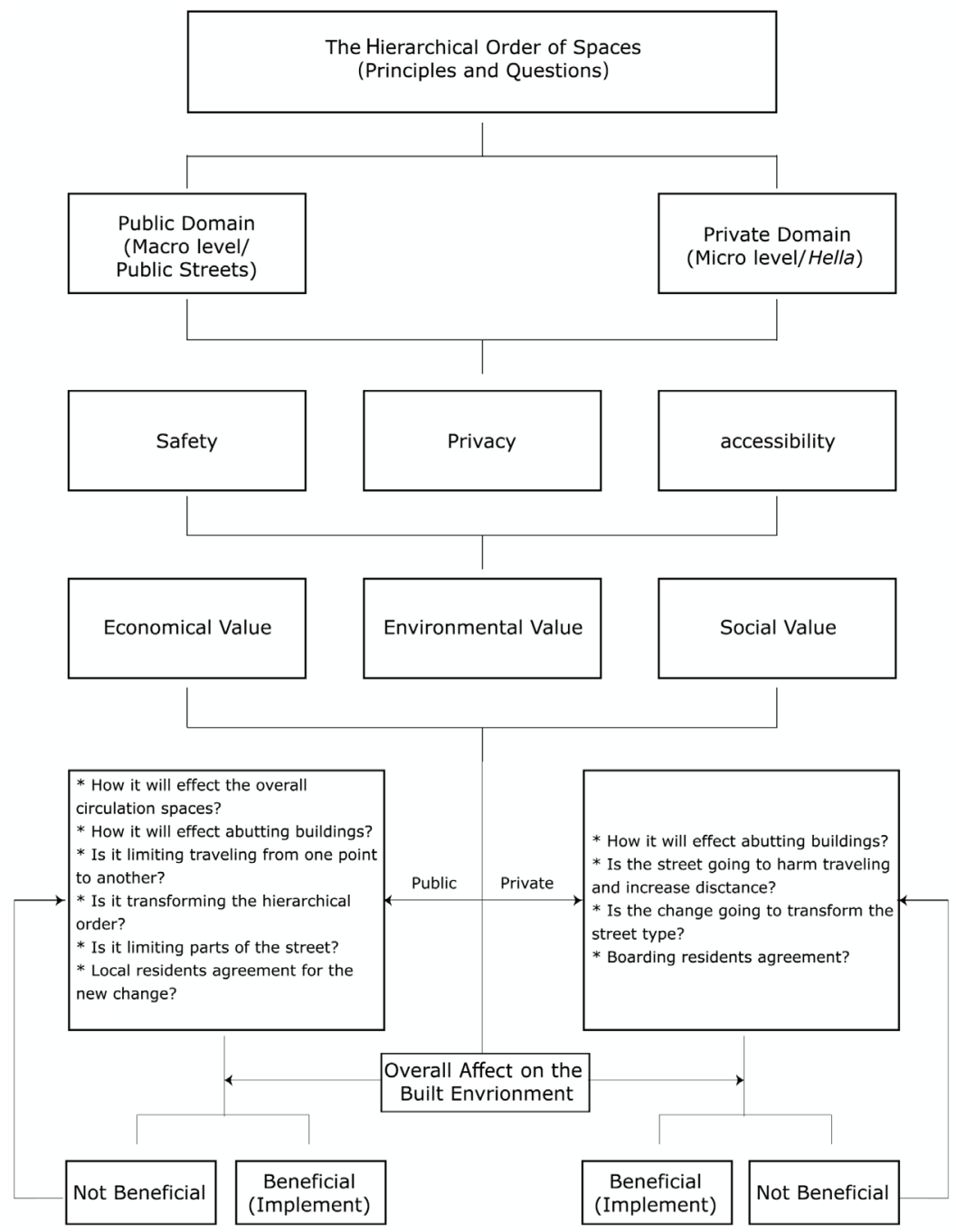

Figure 2. Scenario for proposed principles and questions at the macro and micro level in the decision-making process of the streets hierarchical order. Source: Author.

order of spaces that is guided by the core concepts embedded in the spatial order. The core concepts are the shared and agreed upon tools by which people produce meaningful forms.

It is mentioned throughout this discussion that public streets usually resist change as they require community agreement, while semi-private streets (or pathways) are more flexible and allow more changes to occur. This flexibility on the part of semi-private streets is because they are part of the middle social circle which requires agreement among the neighborhood's inhabitants. The process of street production within the principles of the hierarchical order of spaces allowed people to generate different shapes of streets during the growth of the settlements. This means that people determine the shape and size of the street according to the proximity of their buildings. As a non-binding but guiding principle, public streets should not be less than seven theraa (almost 3.5 meters), while the semi-private street is less and depends on internal agreement among the hella inhabitants (Figure 3). 


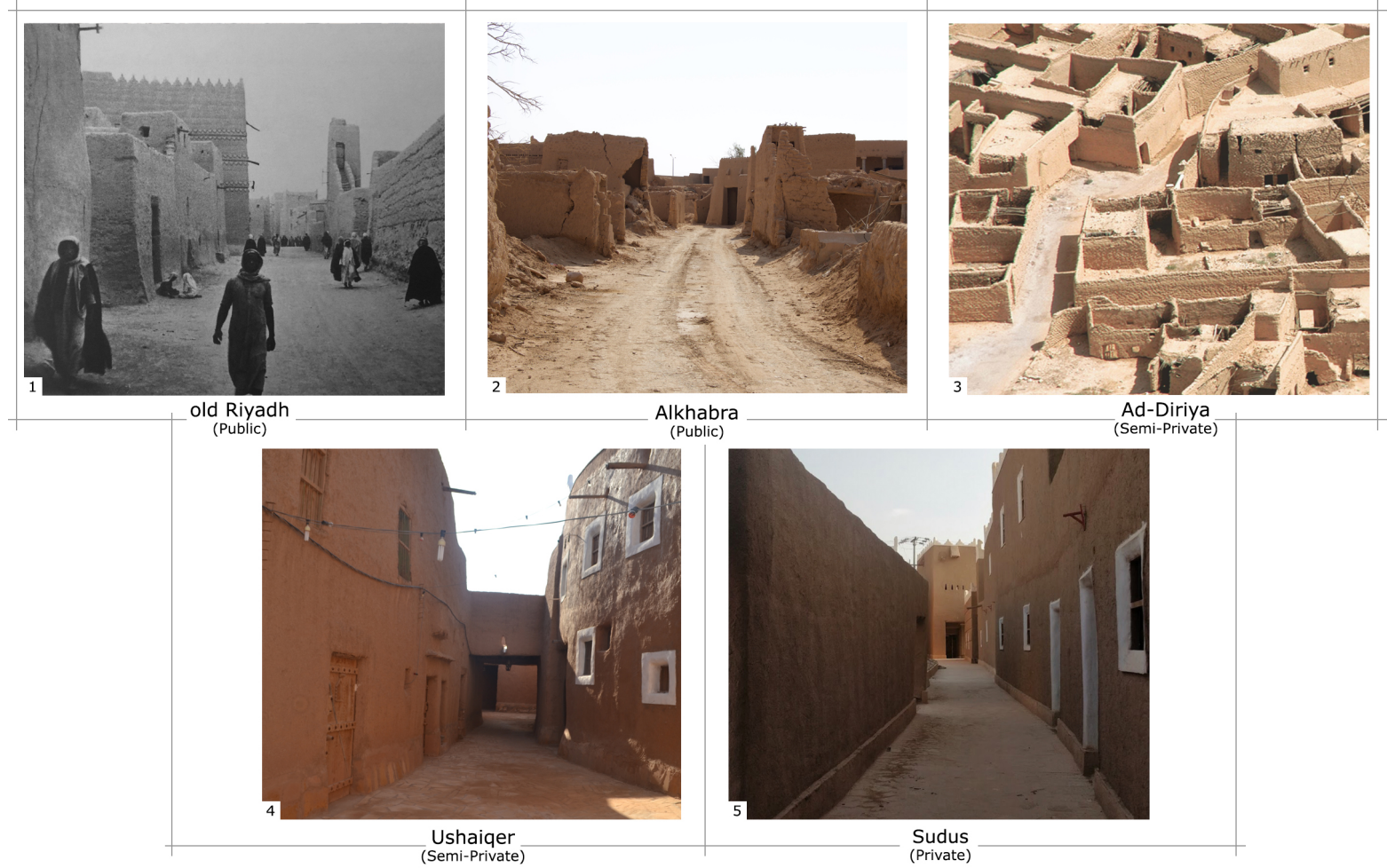

Figure 3. Different external space types. Source: $(1,3)$ ADA, $(2,4)$ Author, and (5) Ibrahim Abdulaziz.

This study uses the Space Syntax Axial Line map technique to examine the integration and connectivity of two types of streets ${ }^{5}$. The integration maps help to understand how the settlements generate the two street types and their hierarchy, while connectivity maps are used to examine how the streets and pathways are interconnected and how they are controlled by the hierarchal order of spaces. These maps take into consideration the values that encouraged the street types to generate as they did, such as economic ability, local resources, environmental conditions, and socio-cultural values. The goal here is to understand why the traditional Najdi built environment needed street classification, and to what degree street classification influenced the process of making the built environment.

We examined and identified exterior space patterns in the five case studies. The study found that public streets were usually well-defined and linked directly with the main gates and with the main public space (the center), while semi-private streets were narrower and deeper inside the hellas with more irregular patterns. The first difference noticed between the two street types is that in the public type, the streets are continuous throughout the urban fabric and usually linked with semi-public passageways. In contrast, in the semi-private ${ }^{5}$ Space Syntax is a useful method for understanding a space or the hierarchy of a space. It can reveal the social meaning of the spatial system and the purpose of buildings [14] [15]. The purpose of an axial map is to represent directions of uninterrupted movement and visibility. In an axial map, a set of lines intersect and cover an entire free space which then comprises a map of lines. See more [14] [16]. 
street type, the pathways typically start with a semi-private pathway that connects, on one end, with a semi-public passageway toward the public space and from the other end with the private spaces in the cul-de-sacs and the main door hallways of the houses.

Examining the two types of streets, another subclassification in the semi-private street (or pathway) type is found that discourages a direct link between the most public with the most private spaces (Figure 4). This is why it was rare to find a cul-de-sac connecting with a public street. If this happened, it is possible to say that it was because of patrimony (death of house owner), where usually the houses and the connecting cul-de-sac transformed and connected with other street types, which reproduced a new street configuration.

The street classification analysis shown in (Figure 6) supports the study's argument that the public street type at the macro-level, and the private pathway type at the micro-level define the location of thresholds. These thresholds define the boundaries of the hierarchal order of spaces and represented as points of separation, gradual transition, and most importantly, as a level of depth between public and private domains.

\section{Street and Space Patterns}

The spatial order produces different patterns and shapes of streets and open spaces. This is because streets and open spaces generated from the decisions taken by people at macro and micro levels, which mainly depend on how they reach an agreement without causing any harm to anyone of the residents. These decisions produced different shapes for the streets and open spaces. Buildings define the shape and boundaries of these spaces which, in general, are unplanned and, in many cases, depend on the principle of Alahyaa, referred to as "reviving the land". As the value was in the building, not in the land, it is for this reason that houses differ in sizes and shapes. The process of Alahyaa principle deeply influenced the patterns and shapes of the streets and open spaces because it created the irregularity of the spaces.

The hierarchal order of spaces in the traditional Najdi settlements usually respects this irregularity and follows its organic results and continuously reproduces the hierarchy and delineation between the public and semi-private streets by generating hidden boundaries (thresholds). The principle of Alahyaa encouraged people to develop their built environment according to their social and economic needs. This is why the urban mass composed of small and large irregularly shaped houses which accordingly define the inner streets and open spaces is seen in the settlements. This, in fact, goes along with the fundamental concepts of the easement right principle that operates in parallel with the Alahyaa principle to achieve "no harm" to anyone.

${ }^{6}$ Reviving the land is based on the principle of freedom of action that Islamic law provides to individuals. Alahyaa as a principle of Islamic law of property whereby a person can acquire ownership of land from the State by way of rehabilitating or bringing back to life dead (mawat) land. See more [17]. 


\section{THE TWO STREET TYPES CLASSIFICATION}

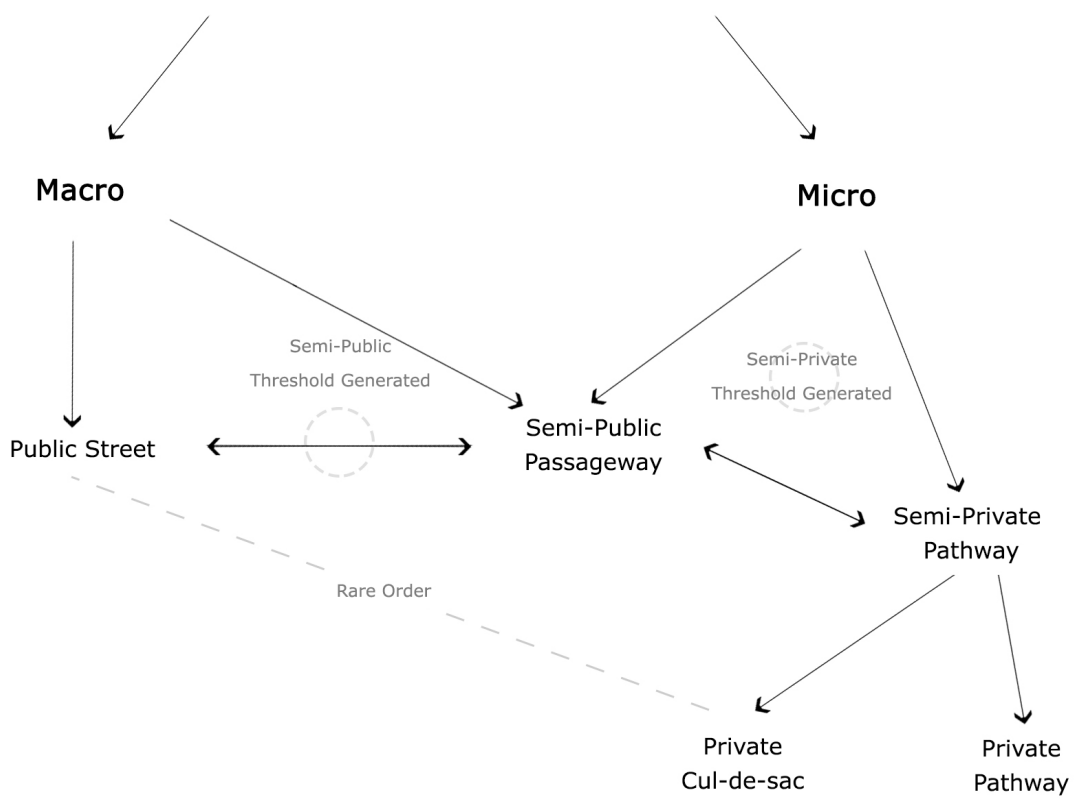

Figure 4. The street types classification in Najdi settlements. Source: Author.

As discussed earlier, a number of principles, such as Alshuf a (neighbors' right), Haq Alirtifaq (easement right), the principle of no harm, and Alahyaa (reviving the land) were used as mechanisms to regulate and manage the dual decision-making process at the macro and micro levels. This is because there was no written law and in most of the Najdi settlements, the head of the community was among the common people, which made these religious principles develop with maximum flexibility in their interpretation to allow people to adapt to their particular social and economic circumstances. In general, the street pattern that resulted from these processes was organic but reflected the deep bond between people and their built environment.

\subsection{Public (Macro) Street Pattern}

Clan arrangements, shared beliefs, and women's role in society strongly influenced the degree of integration or separation in the hierarchal order of space formation. This is why two street types that operate at macro and micro levels were necessary to accommodate such strong influences. In public streets, inhabitants practice most of the public activities, which make them high density, circulated spaces compared to the semi-private ones. Typically, public streets are linked to main gates, and feed to the most active public areas such as the souq.

Public streets are less likely to change as any change requires the agreement of the entire society. This is because they are not only considered the most active spaces but are also spaces that are mainly used to connect different parts of the built environment (Figure 5). In this sense, the public street type is at the macro-level and typically it is controlled by the head of the community, who re- 
sponds to the collective agreement of the settlement residents to ensure adequate circulation flow. In fact, the four main religious generative core concepts discussed earlier are under more communal pressure here and are less flexible because they lie in the larger social circle, which requires more extensive communal agreement. This is why most of the public streets are semi-regular in shape as the mechanisms that generate irregularity in the semi-private streets are less active in the public domain.

By examining the streets at the macro-level, we observed that they generate, to some extent, semi-regular patterns that expand from the center to other areas. While public streets are integrated with semi-public passageways, this integration established a continuous flow and connectivity among different parts of the built environment (Figure 5). In this sense, the semi-private pathways that are usually associated with private spaces are a step away from the public streets. As a result, the connection identified between the semi-public passageways and the semi-private pathways generated a hidden element called aataba (threshold) (Figure 9(B)). It is based on how people reacted and interpreted the hierarchal order of spaces. This makes formation of the aataba a necessary element (mainly non-physical) that the people generated to have space with the flexibility to separate the direct linkage between the public and private spaces. The aataba formation also extends the transition and creates a gradual depth that is evident in public, semi-public, semi-private, and in private areas.

The public street is wider, semi-defined, and feeds into the center of the settlement (souq) (Figure 6(A)). Buildings that are located on this street type are typically owned by Tujar (merchants), which requires that these buildings have more than one entrance on the ground floor, with one entrance to accommodate public access to small shops as well as an additional entrance or entrances to provide private access for one or more families residing in the building (Figure $6(C)$ ).
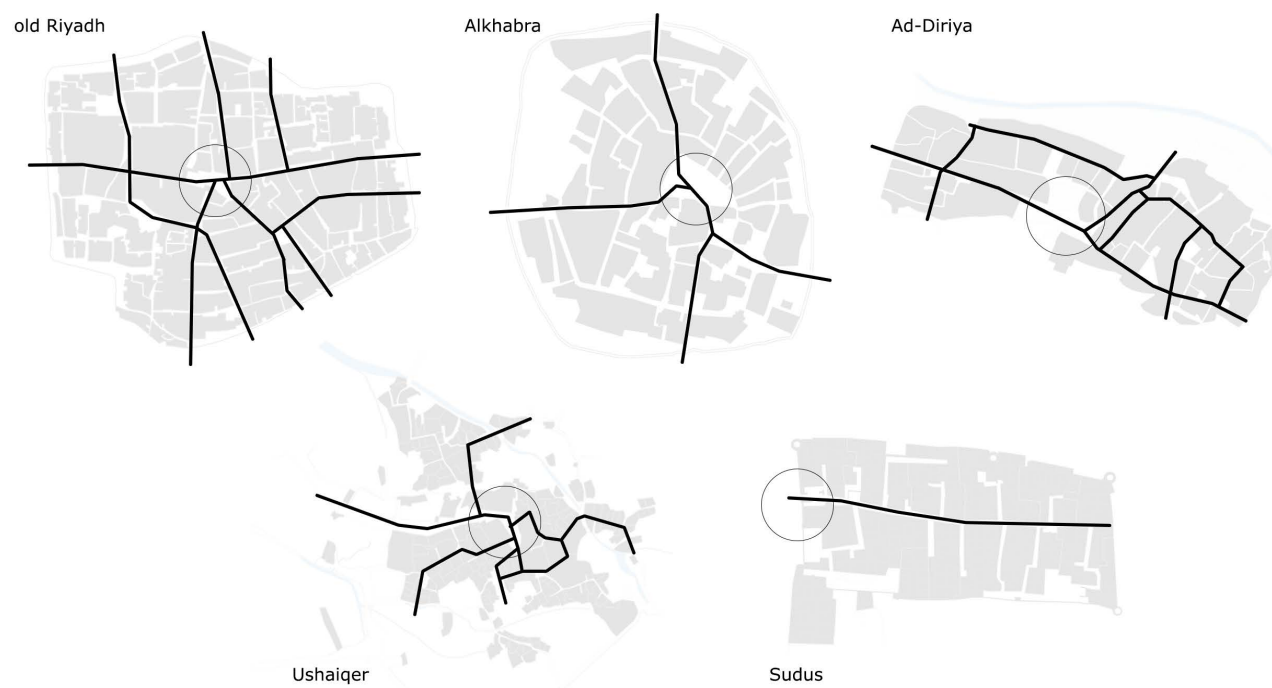

Figure 5. Patterns of public streets in the traditional Najdi settlements. Source: Author. 


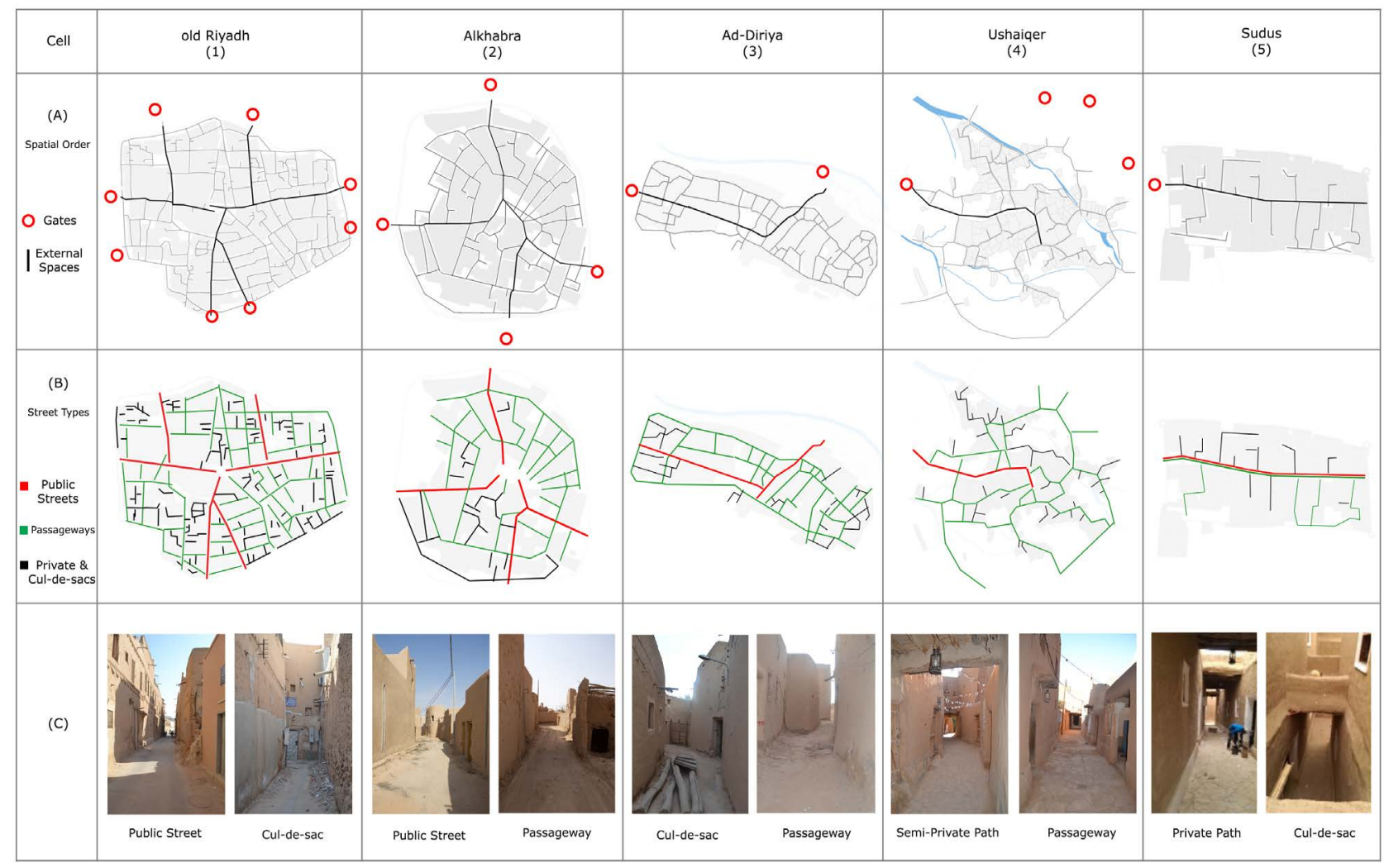

Figure 6. Public and semi-private streets and their different patterns in the traditional Najdi settlements. Source: Author, Sudus images by Al-Saud, 2017.

The argument here is that public streets are usually more oriented toward commercial needs; thus, the settlements assign high priority to commercial use through the higher economic value of the space. This street type is likely associated with different daily life needs and populated by different people, which makes its pattern more extensive and semi-defined. It also supports mixed-use activities by connecting the center of the settlement, where the main commercial and entertainment activities take place away from the private neighborhoods (Figure 6(B)).

According to several interviews between 2016 and 2018, the public street appears to have two functionalities that create different hierarchical orders based on use: the order changes according to time of day, with daytime and nighttime each having their own hierarchical order. In the daytime, gates are open for visitors or travelers to allow them to conduct commercial activities; thus, inhabitants consider this street type at this time of day as having a separation between public and private spaces. In nighttime and when the community closes the gates, settlement occupants are the only occupants of the public streets; thus, the street type transforms to a semi-public transitional space between different hellas.

According to several interviews between 2016 and 2018, the public street appears to have two functionalities that create different hierarchical orders based 
on use ${ }^{7}$ : the order changes according to time of day, with daytime and nighttime each having their own hierarchical order. In the daytime, gates are open for visitors or travelers to allow them to conduct commercial activities; thus, inhabitants consider this street type at this time of day as having a separation between public and private spaces. In nighttime and when the community closes the gates, settlement occupants are the only occupants of the public streets; thus, the street type transforms to a semi-public transitional space between different hellas.

For example, an interview with the local community of Alkhabra in 2018, indicated that the local people developed a semi-public passageway that connects different private parts of the urban fabric ${ }^{8}$. The significance of these passageways, as described by the locals, was to ensure that local people did not have to interact with visitors or travelers that were making use of public streets or spaces in the daytime (Figure 7). The spatial order guaranteed maximum flexibility for the residents to move between and among their private neighborhoods and let the whole center of the settlement be used for commercial activities, which led public streets to be excessively populated by local and outsiders' merchants and their customers in the daytime. The semi-public passageways, then, were essential in the daytime for the local community to ensure low traffic spaces and less public engagement.

By examining the street pattern of Alkhabra, it is found that the semi-public passageways are usually connected with public streets near the main gates, and in addition, they do not lead to private spaces (Figure 7). The semi-public passageway pattern is circular and loops around the urban fabric that leads from one public space to another. This pattern provides inhabitants with the option to enter a semi-private pathway, avoiding the public streets. The level of integration seen in the Alkhabra settlement gave the local community the ability to preserve their private space and traverse the settlement without the need to use public streets. This type of generative process in streets is also evident in the other four Najdi settlements, which helped to understand and appreciate the essential role of primary and secondary gates in the use of the urban fabric. The dynamism in the hierarchical order of spaces uncovered several values implemented by local people in the public street type to support their way of life and their needs.

\subsection{Semi-Private (Micro) Street Pattern}

The previous discussion, we analyzed the street types and identified that the semi-private (micro) pathway type primarily contains two subclassifications as part of the spatial order. A semi-private pathway is considered a semi-private right-of-way path, while a private path or cul-de-sac, according to Islamic law, is private property with ownership limited to the people who live in the houses accessed by it (Figure 8(B)).

${ }^{7}$ Focus group interviews conducted in Alkhabra, Ushaiqer and old Riyadh in 2017 and 2018.

${ }^{8}$ Focus group interviews conducted in Alkhabra on 1/1/2018. 


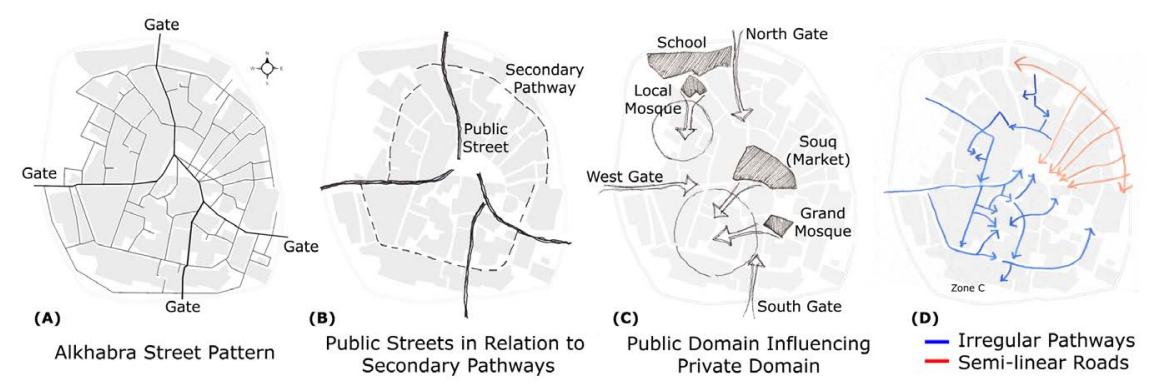

Figure 7. Alkhabra street pattern. The diagram shows the different factors that influenced the shape and pattern of the different types of the street system. Source: Author.

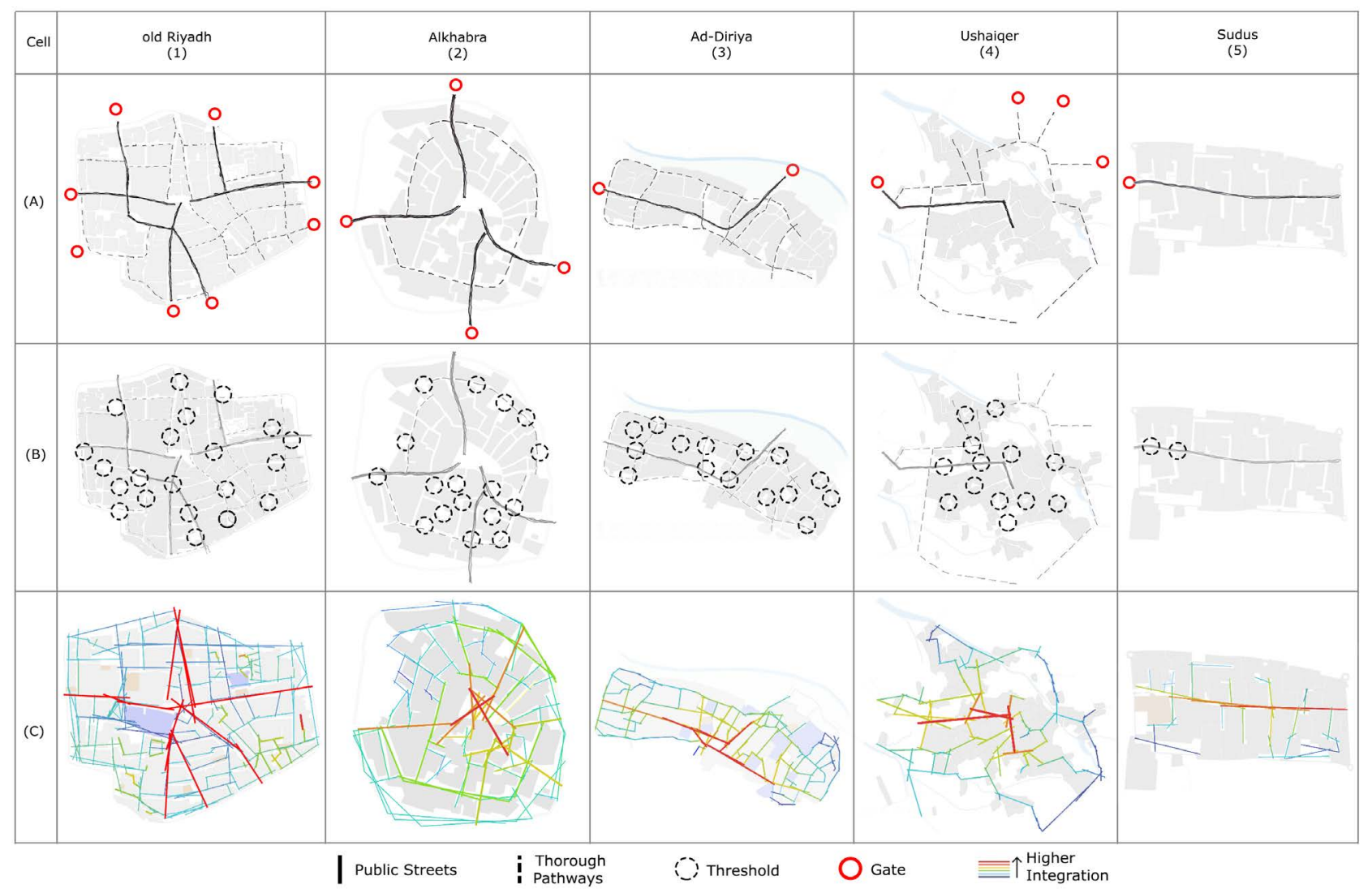

Figure 8. Examining the semi-private pathways in the traditional Najdi settlements. These pathways usually connect with the public streets to create a threshold and branch into private pathways inside hellas. Source: Author.

The subclassification of the street types establishes a measurement of how much input people can have on the private pathway type. Even though this study mentions earlier that the private pathway type is owned by the private domain sub-collectively, the semi-private pathway is usually generated to provide a link between different hellas and to connect a hella with public and semi-public passageways. The decisions that take place in this semi-private domain and the middle social circle are wholly linked, which allows for more flexible changes by individuals because it is limited to the agreement of the neighborhood's inhabitants. The principle of "no harm" is crucial in these areas and inhabitants always apply this principle to evaluate and judge a proposed change, but usually it is 
more likely to implement a proposed change in these areas than elsewhere.

The cul-de-sac or dead-end road is a private pathway which emerges in two ways, either as a spontaneous formation by a group of individuals or it may emerge over time as a result of the easement right due to a division of the houses either due to selling part of a house or due to patrimony (death of the house owner). This type of external space is considered to be privately owned by a specific group of people, a group which has complete control of the space. As a result, the space is more flexible and can change or disappear at any time if the owners decide, for example, to sell their houses and reconfigure the space into one big house. It is flexible space as the radius effect to any change in this private pathway will not go beyond the residents occupying it. Jamel Akbar argues that the private pathway is isolated and not still well-defined as a semi-private pathway; thus, people will utilize, modify, or reconfigure it to be appropriate to the surrounding residents [8].

Examining the semi-private street types, it helped to develop an understanding of how the semi-private and private pathways generated based on the hierarchical order of spaces. We broadly noticed that semi-private pathways usually are not located in deeper private areas, which makes it possible for them to connect with public or semi-public streets. However, the semi-private pathways were generated to establish a gradual link between the internal domain (hellas) where the private pathways are located, and the external domain (souq) were public streets are found (Figure 9).

Using the Space Syntax Integration map technique integration here means the act of combining a single element into an integrated whole. By referring to (Figure $8(C)$ ), it seems that the private (micro) street pattern forms a gradual integration with other street types, especially if the street is public. This study identified thresholds where a semi-private pathway connects with public or semi-public streets. The argument here is that these thresholds are generated to create a gradual transition and to separate public streets and private pathways (Figure $8(\mathrm{~B})$ ). In this sense, the semi-private pathways can extend across the built environment to establish a link between different hellas as well as to connect with the public streets, while the private pathway usually emerges from the semi-private ones (Figure 8(B)). It is found that in the Sudus settlement, where it is considered, as a whole, to be a single, private domain and the majority of the public activities are conducted outside the built form boundaries of Sudus, the hierarchal order of spaces identified is less in terms of depth; however, as a whole, it has a similar generative process as the other four cases.

Therefore, the process of control determines the hierarchical order of spaces in the traditional Najdi settlements. The study observed this when specific users controlled the private pathways in the traditional Najdi built environment, and the accessibility to those spaces required a gradual transition, which makes it difficult to reach in most cases. The entire community controlled the public streets, which meant that there was no restriction in the locals using them. This is similar to Reference [18] note when he describes that controlled spaces "Gives 
the controller a space to use at his discretion. It gives him freedom within its boundaries to arrange forms as he pleased" [18]. In this sense, the hierarchical order of spaces is not meant only to control the connectivity and integration of street types but it is also meant to establish a gradual depth in the traditional Najdi settlements to create several controlled points for the community to engage with the placemaking process without effecting the solidarity of spatial order (Figure 10(B) \& Figure 10(C)).
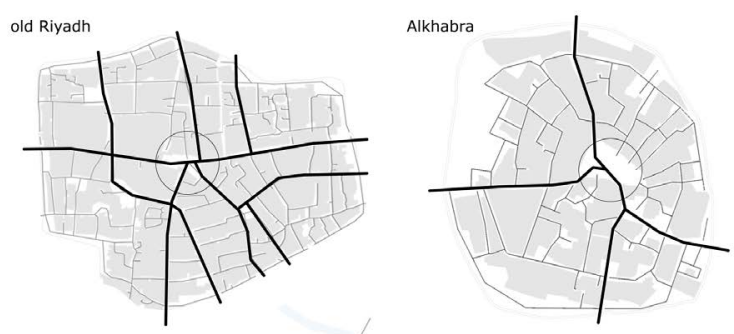

Ad-Diriya
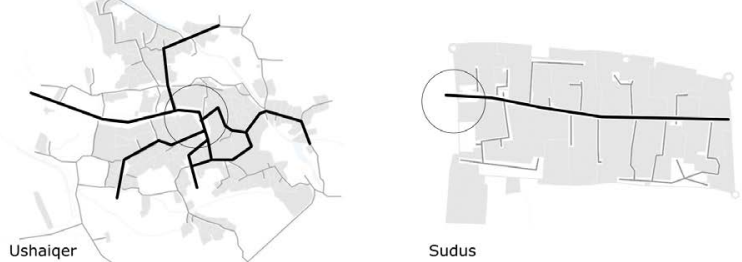

Figure 9. Micro street type pattern in the traditional Najdi settlements. Source: Author.

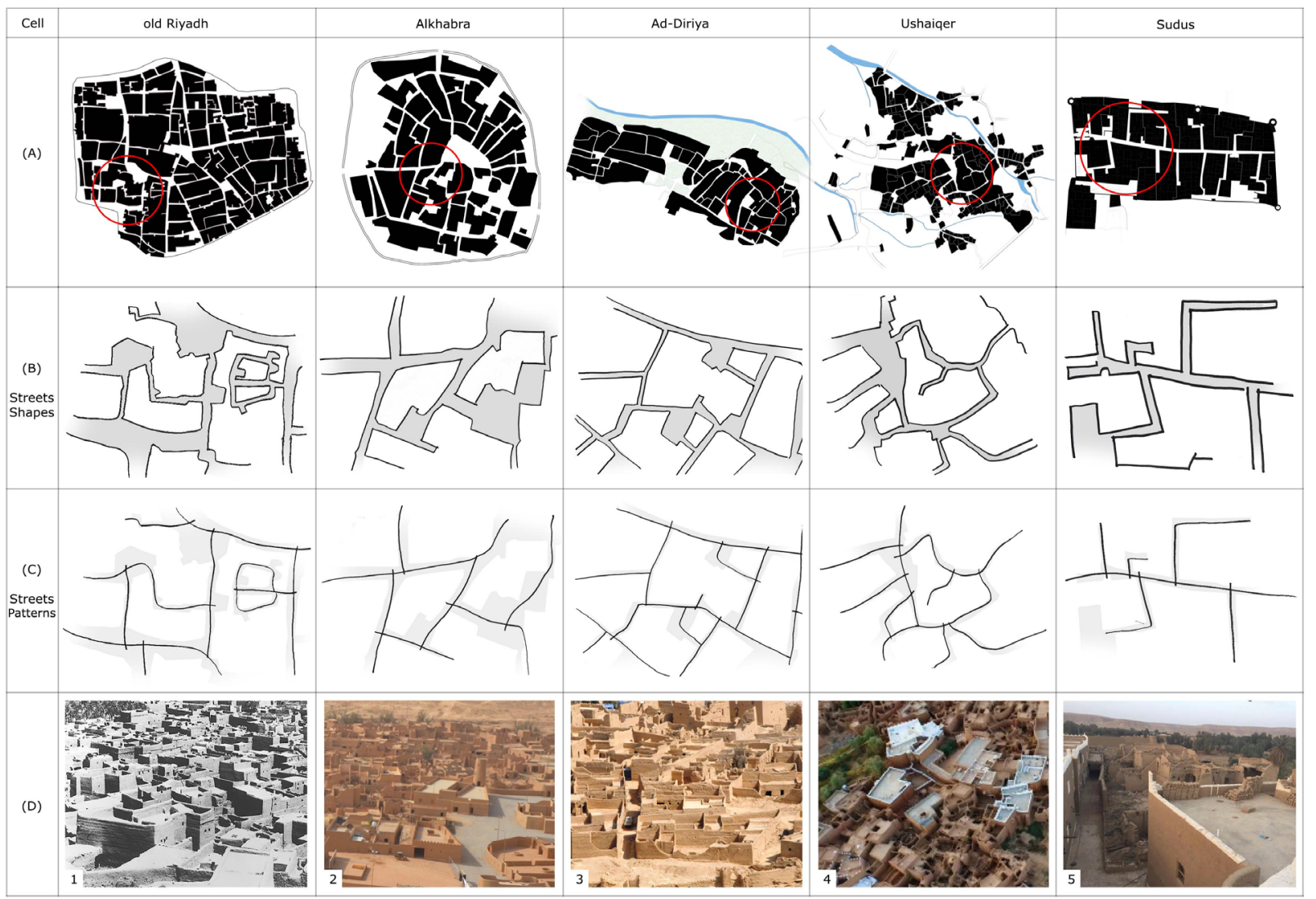

Figure 10. Street and open space shapes and patterns. Image Sources: (1, 3 and 5) ADA, (2) SCTH, (4) Al-Arabiya Magazine. 


\section{Findings and Discussion: Shapes and Forms of External Spaces}

How the patterns and shapes of streets and spaces are an organic product of people's decisions is discussed and explained through their implementation of the four religious and social principles of Alshuf a (neighbor's right), Haq Alirtifaq (easement right), the principle of doing "no harm", and the principle of Alahyaa ("reviving the land"; that is, the encouragement to build according to social and economic needs). Although people were conscious of their decisions, the urban physical shapes were a direct result of these decisions without the existence of any preconception of the shape. The interesting thing is that people knew that the resultant shape, even if it was irregular, was in harmony with the rest of the built environment. This study considers this process as one of the main embedded mechanisms of the spatial order.

By examining the hierarchal order of spaces and its two street types, we identified different shapes and forms for how streets generated. Inhabitants produced and reproduced these organic shapes based on their inherited cultural values and building know-how to satisfy their functional requirements, social needs, and personal preferences. According to interviews conducting between 2016 and 2018, some street shapes were formed to signify which type of street the space belonged too, and they were produced in a particular way to break the continuity of different connected street types. In a few cases, local people decoded the transition between street types by being alert to specific shapes that signified that the street type has changed.

In the semi-private pathway, the pattern of the path is irregular. This irregularity is because the path is not meant to segregate spaces, but it is meant to define them and classify several hellas to social groups within that particular private domain (Figure 11). This is why the semi-private pathway takes the shape of an irregular pattern and why it varies from medium to narrow width. In contrast, a private cul-de-sac has somewhat different characteristics and its form depends on which activities are close by, such as its proximity to the semi-public street, and how it is connected with a semi-private pathway.

For example, in the Ushaiqer settlement, an architectural element exists called Mujabab. This element is a closed structure that appears within a corridor that cuts between two facing houses. Running below the Mujabab architectural element is a medium to narrow semi-private pathway (Figure 12). The element is mostly an internal functional component of the house; however, its external physical presence on the street affects the road, causing visual discontinuity, affecting social activities on the street, and impacting the environmental quality of the street, as well as affecting the external appearance of the house?

${ }^{9}$ Mujabab is not considered a pure architectural element originated by Najdi inhabitants. This element appears in a number of Arabic Islamic traditional cities (called "Sabat") such as Cairo, Tunisia, Damascus, Jerusalem, etc. However, it is important to note that the Najdi inhabitants developed it in a unique way to accommodate and fit their particular socio-cultural needs [8] [9] [19]. 


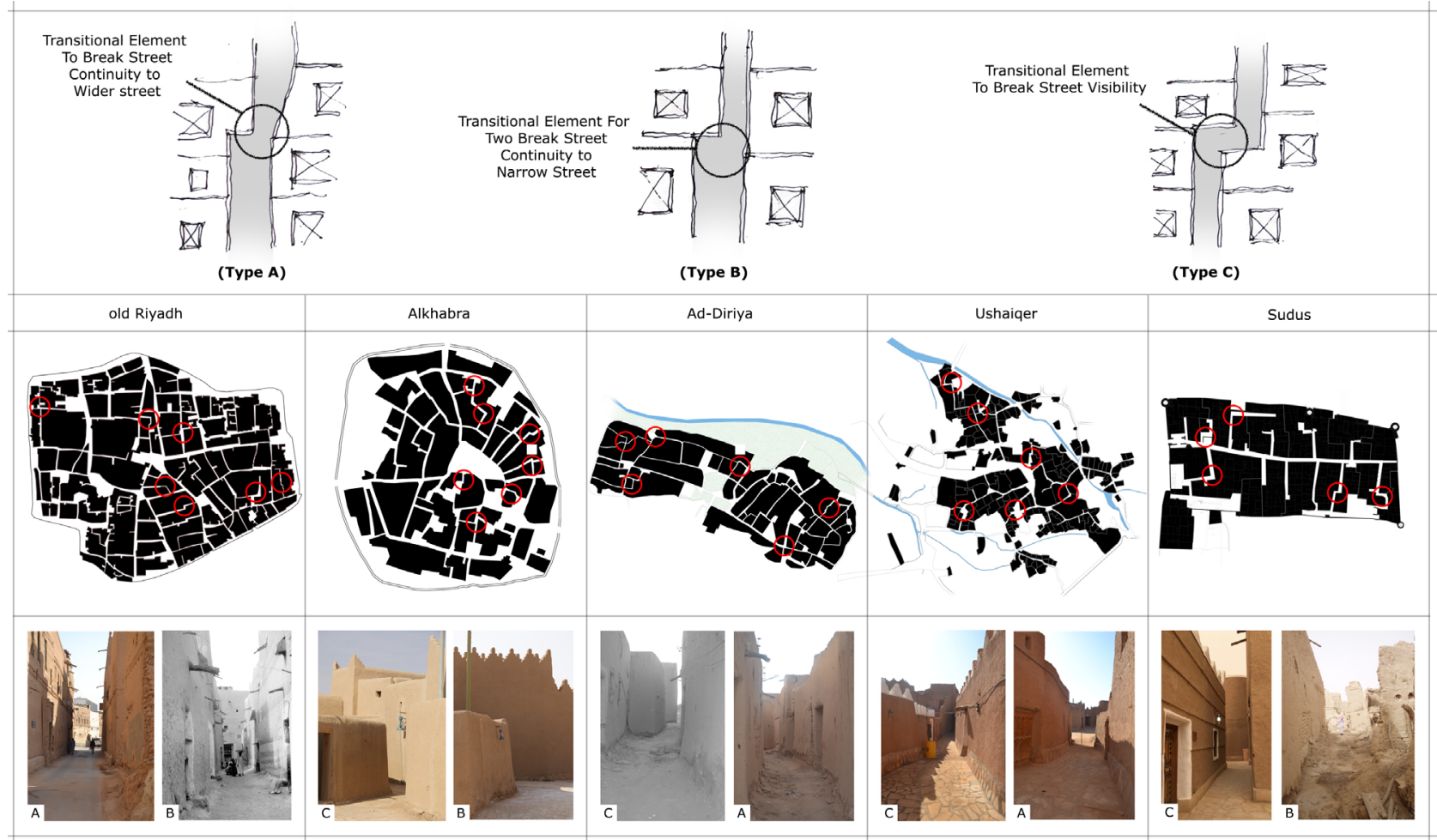

Figure 11. Formation of the roads and how sometimes street shapes signify a change of street type and a change in usage.

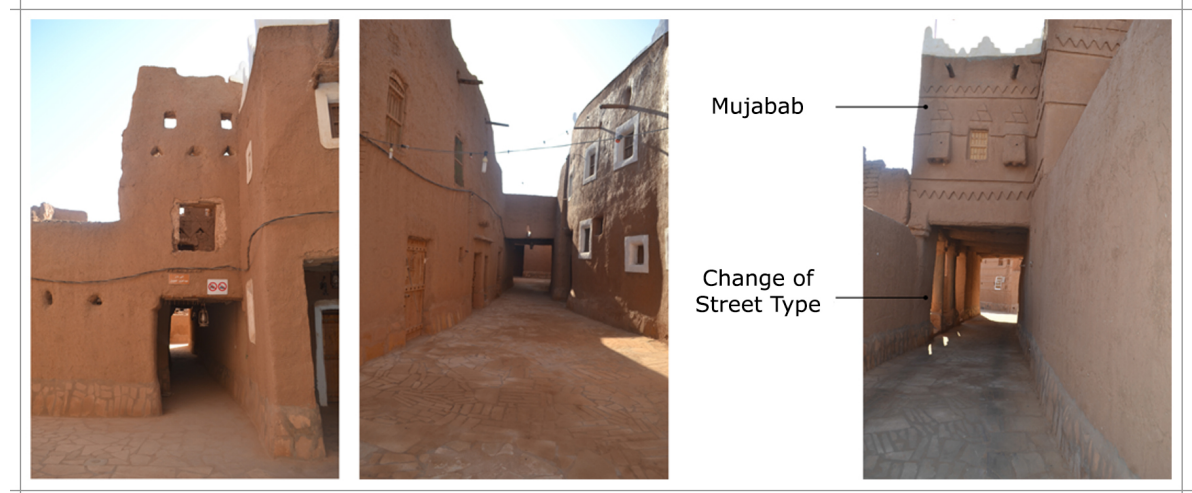

Figure 12. The different implementations of mujabab in Ushaiqer, (left) the mujabab is used to separate public and semi-private pathways, (middle) it is used as transition space, (right) it is used in a way that can be used as a gathering spot for social activities. Source: Author.

At the physical level, a Mujabab can serve as a visual symbolic element to signify that the street type has changed from semi-public to semi-private or semi-private to private. The Mujabab socially can function in different ways for different inhabitants, such as a threshold element by local people to signify a controlled area, to signify a transitional space between different hellas, and as an element to break the discontinuity of two street types visually. Since the Mujabab impacts the discontinuity of varying street types, the produced space functions as a social gathering spot between different hellas. Inhabitants understand the 
different location of the element and its associated meanings within the built environment. At the environmental level, the Mujabab provides a shaded area for people to socialize away from direct sunlight beams. Local people decode the meanings that the Mujabab contains based on how the element is placed, especially in a harsh climate like the Najd region, which encourages people to use the $M u j a b a b$ as an outdoor gathering space for social interaction.

However, the shapes produced in the external spaces are not necessarily physical. In this regard, we observed that in the Ushaiqer settlement the irregular shapes of the streets serve to signify a change of street type. These shapes were mostly evident and produced in the private (micro) type. Dead-end paths are characteristic of this type, while the length and width of the pathways are always dependent upon who occupies the space along the path (Figure 13).

The argument here that chowks or blind dead-end paths are products generated by locals to reflect their socio-cultural needs in the space. Locals interpret this reflection differently, as they understand that different spaces are connected and arranged based on the hierarchal order of spaces which leads to a specific accommodation to overcome certain issues. Therefore, the process of making in particular places is significant and evidence of local people making a contribution to the placemaking process in the built environment. This led to a strong attachment to particular places, as it gave the individual the necessary values to maintain such a place or space for an extended time.

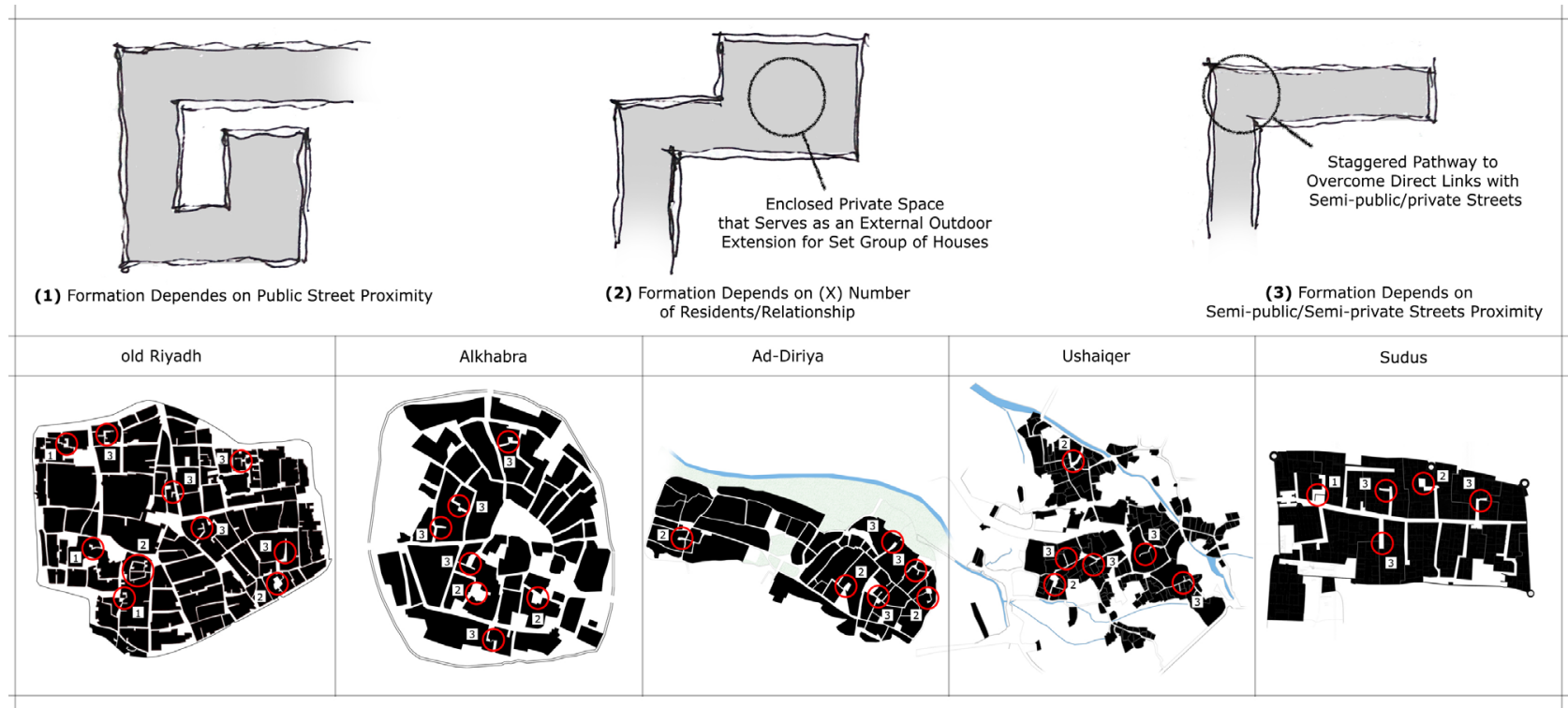

Figure 13. Formations of chowks and blind dead-end paths in the traditional Najdi settlements. Source: Author.

\section{Conclusion and Recommendation}

This paper describes how the hierarchal order of spaces emerged to ensure a smooth transition between public and private domains, and how its inhabitants had the ability to influence the placemaking in particular places. The analysis of 
the street network helped to reveal how strongly the streets and pathways affected the organization and the quality of the traditional built environment. This led to several developments of urban mass patterns and ensured that each mass is different from the others. The development of the hierarchal order of spaces is a mechanism for protecting the social order and representing the values and lifestyle preferences of the people. As a result, the local community interpreted the process of making the built environment in different ways by using the hierarchal order's four principles to meet their physical needs. This resulted in urban masses that have different spatial organizations and forms, but produced similar spatial orders and served similar functions.

The two levels of the decision-making process at the macro and micro levels increased the possibility for the community to be involved in the concept of placemaking. This is because at the macro-level, the impact was minimal, and inhabitants made most of the decisions that shaped the traditional Najdi built environment and maintained its existence at the micro-level. This, in fact, produced a very similar, but not identical process of generating spaces and forms across the five cases.

The Hierarchal Order of Spaces can be seen as a core concept to how to generate a coherent built environment. Examining the spatial order of particular place may identify how street types and their different shapes and forms were the tools that people used to show how they interpreted their shared religious and social principles to produce their own hierarchal order of spaces. It may also show us why different places need different approaches to how we generate their spaces as different groups of people require different needs and accommodations.

Future studies can test and examine current spatial orders in modern cities or particular places within the cities to observe and reflect how a built environment generated sub-identities and how certain places have significant value than other places. These findings may help the future city-wide vision of urban planning create better spaces and accessibility and, more importantly, privacy while maintaining the coherency of the urban fabric. This, we believe, can uncover several issues related to how and why different parts and places seem neglected or separated from the holistic network that a city established for itself.

This study can expand the debate for urban planning practice, and the concept of placemaking in the Saudi Arabian context as both areas are seen as a synthesis of how a built environment can be homogenized, which is a rare perspective in Saudi Arabia. This paper provides and highlights the importance of understanding the context in order to address social and cultural issues to generate a productive, built environment.

\section{Acknowledgements}

The author would like to thank Saudi Arabia governmental entities, and all other individuals for their support and sharing the data, which improved the quality of survey output. 


\section{Conflicts of Interest}

The author declares no conflicts of interest regarding the publication of this paper.

\section{References}

[1] Norberg-Schulz, C. (1981) Genius Loci, towards a Phenomenology of Architecture. Rizzoli International Publication Inc., New York.

[2] Richardson, M. (1982) Being-in-the-Market versus Being-in-the-Plaza: Material Culture and the Construction of Social Reality in Spanish America. American Anthropological Association, Arlington, 421-436. https://doi.org/10.1525/ae.1982.9.2.02a00120

[3] Ralph, E. (1976) Place and Placelessness. Pion Press, London.

[4] Lefebvre, H. (1991) The Production of Space. Blackwell Publishing, Hoboken.

[5] Cresswell, T. (2004) Place a Short Introduction. Blackwell Publishing, Hoboken.

[6] Cresswell, T. (2008) Place: Encountering Geography as Philosophy. Geography (Sheffield, England), 93, 132-139.

[7] Agier, M. (2015) Anthropologie De La Ville. PUF, Paris. https://doi.org/10.3917/puf.agier.2015.01

[8] Akbar, J. (1981) Responsibility and the Traditional Muslim Built Environment. Unpublished Ph.D. Thesis, Massachusetts Institute of Technology, Cambridge.

[9] Hakim, B. (1986) Arabic-Islamic Cities: Building and Planning Principles. Kegan Paul International, London.

[10] Alajmi, M. (2009) History of Architecture in Kuwait: The Evolution of Kuwaiti Traditional Architecture Prior to the Discovery of Oil. Unpublished Ph.D. Thesis, University of Nebraska, Nebraska.

[11] Al-Hathloul, S. (1981) Tradition, Continuity and Change in the Physical Environment: The Arab-Muslim City. Unpublished Ph.D. Thesis, Massachusetts Institute of Technology, Cambridge.

[12] Al-Hussayen, A. (1996) Women and the Built Environment of Najd. Unpublished Ph.D. Thesis, University of Edinburgh, Edinburgh.

[13] Hillier, B. and Hanson, J. (1984) The Social Logic of Space. Cambridge University Press, London. https://doi.org/10.1017/CBO9780511597237

[14] Hillier, B. (2005) The Art of Place and the Science of Space. World Architecture, 11, 96-102.

[15] Jiang, B. and Claramunt, C. (2002) Integration of Space Syntax into GIS: New Perspectives for Urban Morphology. Transactions in GIS, 6, 295-309. https://doi.org/10.1111/1467-9671.00112

[16] Ul-Haq, S. (1992) Meaning in Architecture: An Investigation of the Indigenous Environment in Bangladesh. Unpublished Ph.D. Thesis, Massachusetts Institute of Technology, Cambridge.

[17] Ar-Rami, Ibn (XV Century) (1995) Al-Ilan bi Ahkam al-Buniyan ed. Al-Atram S. Dar-Ishbiliya, Riyadh, KSA. (In Arabic)

[18] Habraken, J. (1985) The Appearance of the Form. Awater Press, Cambridge.

[19] Al-Hatloul, S. (2010) Arabic Islamic Cities: Legislation as Instrumental in Shaping the Urban Environment. Umran Alulom, Riyadh. (In Arabic) 